\title{
Reconstruction of Air Shower Events Measured by the Surface Detectors of the TAx4 Experiment
}

\author{
Hyomin Jeong $^{a, b, *}$ on behalf of the Telescope Array Collaboration \\ (a complete list of authors can be found at the end of the proceedings) \\ ${ }^{a}$ Sungkyunkwan University, Physics Department, Seobu-ro 2066, Suwon-si, Republic of Korea \\ ${ }^{b}$ Cooperation Center for Cosmic Ray Research, Seobu-ro 2066, Suwon-si, Republic of Korea \\ E-mail: jhminie@gmail.com
}

\begin{abstract}
The source, propagation and acceleration mechanism of ultra-high-energy cosmic rays (UHECR) have been investigated since the first discovery of the UHECRs to solve the mystery of the extremely high-energy universe. The Telescope Array times 4 (TAx4) experiment, which currently consists of 257 Surface Detectors (SDs) and 2 Fluorescence Detector (FD) stations, had been built in Utah, USA in 2019. The TAx4 SDs observe secondary particles in an extensive air shower induced by the UHECR, and we reconstruct arrival direction and energy of the UHECR using the signal intensities and timings measured by the SDs. We present the reconstruction procedure of the UHECR and the preliminary energy spectrum above $10 \mathrm{EeV}$ measured by the TAx 4 SDs.
\end{abstract}

$37^{\text {th }}$ International Cosmic Ray Conference (ICRC 2021)

July 12th-23rd, 2021

Online - Berlin, Germany

\footnotetext{
${ }^{*}$ Presenter
} 


\section{Introdunction}

The Telescope Array (TA) project, proposed to study the ultra-high-energy cosmic rays (UHECRs) and operating in Utah since 2008, has the largest detection area in the northern hemisphere. The TA has two different types of detectors to observe extensive air shower, which includes many secondary particles generated by the interaction between the primary cosmic ray and molecules in the atmosphere. The surface detector (SD) array, which consists of 507 two-layered plastic scintillation detectors $\left(3 \mathrm{~m}^{2} /\right.$ detector) covering a area of $700 \mathrm{~km}^{2}$ with $1.2 \mathrm{~km}$ spacing, detects directly secondary particles in the air shower, while the fluorescence detectors (FDs) surrounding the SD array can measure longitudinal development of the air shower to detect ultra-violet lights from the excited air molecules by the secondary particles in the air shower. The TA collaboration reported the medium-scale anisotropy of UHECRs in 2014 [1]. They found a concentration of UHECRs above $57 \mathrm{EeV}$ in the specific direction. Many interpretations and models have been proposed to find the UHECR origin. However, there is no clear evidence so far. We still do not have enough statistics of the UHECRs to identify the UHECR origin. Since the cosmic-ray flux rapidly decreases as the energy increases, a huge detection area is essential to study the UHECRs.

The telescope Array times $4(\mathrm{TAx} 4)$ was proposed to expand the detection area of the TA by a factor of 4. A total of 260 SDs had been assembled in Korea and Japan, and transported to Utah, USA. These SDs were deployed with $2.08 \mathrm{~km}$ spacing at the north east and south east of the TA SD array during the period from 2019 January to March [2], and the TAx4 SDs have been successfully operated since the end of March. In this proceedings, we will present the air shower reconstruction procedure and the quality cuts to improve the energy resolution and the angular resolution of the TAx4 SDs. Finally, the preliminary energy spectrum measured by the TAx 4 SDs will be presented.

\section{Air Shower Reconstruction}

\subsection{Detector Calibration}

The signal intensity recorded at each SD is converted to the number of detected particles. The signal intensity has temporal variation depending on the environmental temperature and humidity. Therefore, a signal intensity when a single particle (muon) passes through each SD (1 MIP) should be carefully calibrated at short intervals. In order to calibrate the SD, 1-MIP intensity, ADC pedestal histograms, and etc. are recorded at each SD every 10 minutes. It is noted that these calibrations are not fully implemented in this analysis.

\subsection{Pattern Recognition}

In the calibrated data, the charge and arrival timing of the signal, the GPS position of the detector, and etc. are recorded at each SD. An air shower trigger signal is issued when three or more adjacent SDs recording more than 3 MIPs appear within an $8 \mu$ s time window. Once the trigger signal is issued, data from the SDs recording more than 0.3 MIPs is collected. The signals induced by accidental atmospheric muons are also recorded frequently. To exclude such signals by accidental muons, the pattern recognition procedure is applied. At first, this procedure finds the largest cluster of SDs that are contiguous in the space and time. The distance between adjacent two detector should be less than $\sqrt{2}$ detector spacing ( $2.08 \mathrm{~km}$ in TAx4 SDs). Figure 1 shows an 

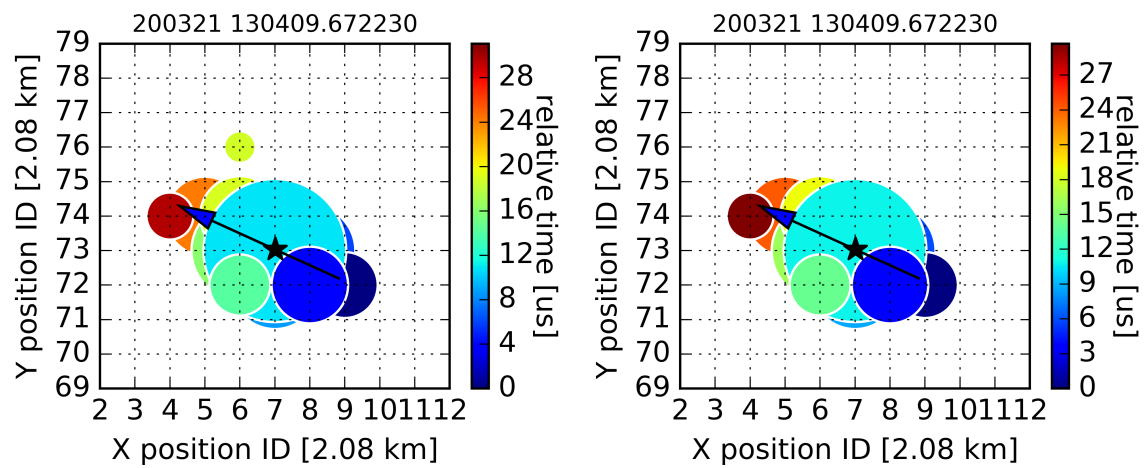

Figure 1: Examples of all the triggered SDs (left) and space-time SD cluster only (right).

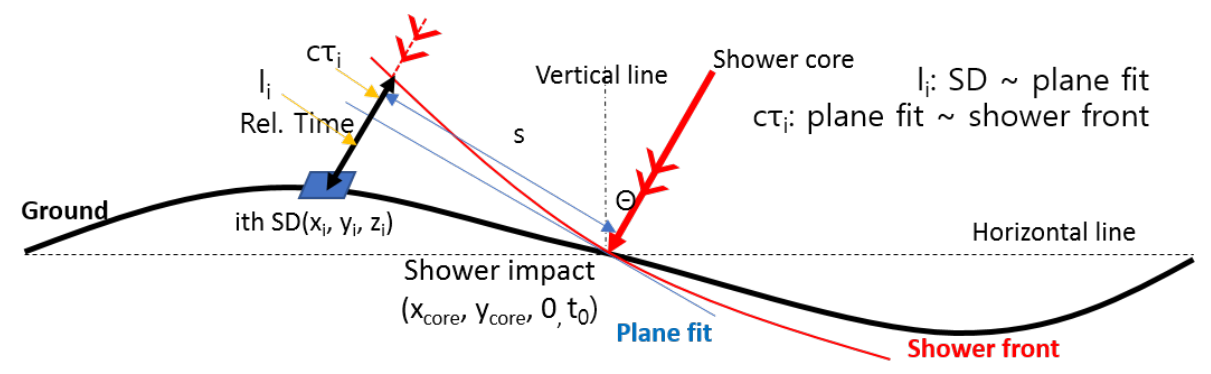

Figure 2: Schematic view of the air shower front.

example of the recorded event display before and after the pattern recognition. A star mark and an arrow in the figure represent the reconstructed air shower core position and direction projected on the ground, respectively. Each circle size and color scale represent the signal intensity and the relative timing, respectively, at each $\mathrm{SD}$.

\subsection{Geometry Fit}

In the geometry fit procedure, we estimate the arrival direction of an air shower using relative timings recorded at SDs. Figure 2 shows a schematic view of an air shower front and the explanation for the determination of the arrival direction. Since the air shower front is not flat plane, the arrival timing recorded at $i$-th SD is expressed as

$$
t_{i}=t_{0}+\frac{l_{i}-z_{i} \cos \theta}{c}+\tau_{i}
$$

where $t_{0}$ is an air shower impact time (timing when the air shower core hit the ground), and $l_{i}$ is a distance from the SD to ideal plane front (blue solid line in Figure 2). $\tau_{i}$ is a time delay from the ideal plane front, and it represents an actual shower curvature. The time delay $\tau$ and its error $\sigma_{\tau}$ are calculated assuming the modified Linsley function.

The core position of an air shower is determined as the center of gravity of signal charges recorded at the SDs. Finally, we obtain the arrival direction of air shower by minimizing $\chi^{2}$ using Equation 1, assuming the determined core position. 

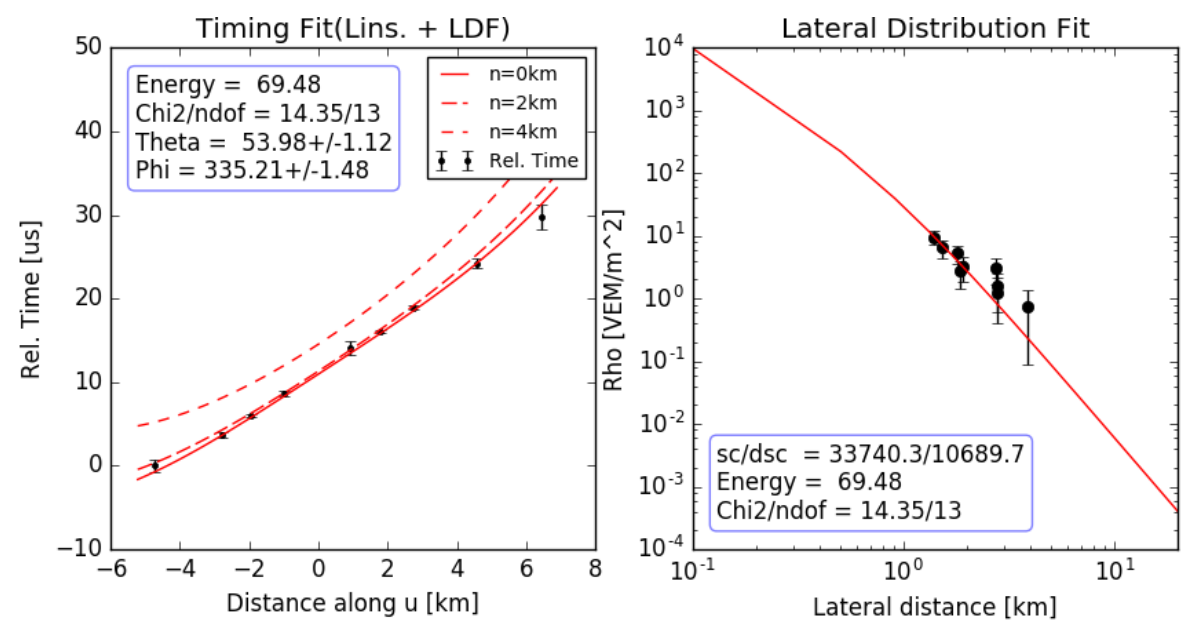

Figure 3: Examples of the geometry and lateral combined fit.

\subsection{Lateral Distribution Fit}

After the geometry fit, we estimate the primary energy of the air shower using the distribution of secondary particle densities in the SD array. The particle density $(\rho)$ as a function of the perpendicular distance $(s)$ from the air shower axis is expressed as

$$
\rho=A\left(\frac{s}{91.6 \mathrm{~m}}\right)^{-1.2}\left(1+\frac{s}{91.6 \mathrm{~m}}\right)^{-(\eta(\theta)-1.2)}\left(1+\left[\frac{s}{1000 \mathrm{~m}}\right]^{2}\right)^{-0.6},
$$

where $\eta$ is

$$
\eta(\theta)=3.97-1.79[\sec (\theta)-1],
$$

taken from previous experiments, the Akeno Giant Air Shower Array [3] and the TA SD array. In this equation, free parameters are the shower core position $\left(x_{0}, y_{0}\right)$ and the scale factor $(A)$.

\subsection{Geometry and Lateral Distribution Combined Fit}

To improve the energy and angular resolutions, we finally apply a combined fit to minimize $\chi^{2}$ of geometry and lateral fits simultaneously. Figure 3 shows an example of the combined fit result.

\subsection{Energy Determination}

Since a particle density at certain perpendicular distance from the air shower axis is proportional to the primary energy, and it can be used as a good energy estimator. In the previous work for the TA SD array, the particle density at a perpendicular distance of $800 \mathrm{~m}$ from the air shower axis (S800) was used as the energy estimator. The conversion factor from $S 800$ to the energy largely depends on the zenith angle of the air shower $(\theta)$. Therefore, the primary cosmic-ray energy is obtained from a 2D plane of $S 800$ and $\theta$. Figure 4 shows the relation between $\sec (\theta), S_{800}$ and the energy calculated by the Monte Carlo (MC) simulation. We generate the air shower MC data using the CORSIKA [4] package with QGSJETII-03 [5] as a hadronic interaction model assuming the primary proton differential spectrum $E^{-3}$. The TAx4 SDs are assumed to be virtual $10 \times 10$ grid array with $2.08 \mathrm{~km}$ spacing. The thrown zenith angle and core locations are less than $60^{\circ}$ and $1 \mathrm{~km} \times 1 \mathrm{~km}$ area of the center of the array, respectively. The estimated energy by the SD array is 


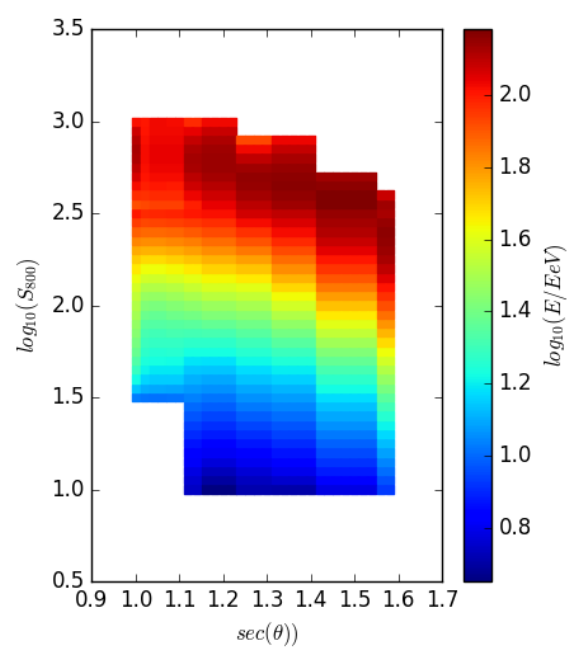

Figure 4: SD energy conversion table
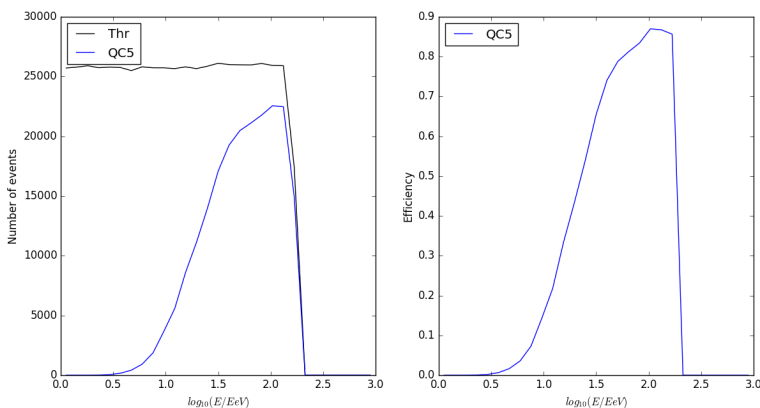

Figure 5: The number of reconstructed events (left) and quality cut efficiencies (right) after the quality cuts

renormalized by a factor of $1 / 1.27$, which was determined in the TA SD array [6], to match the FD energy scale. This normalization factor will be updated using SD and FD hybrid observation of the $\mathrm{TAx} 4$ experiment in the future.

\section{Results and Discussions}

\subsection{Quality Cuts}

In this analysis, we adopt five quality cuts as follows: (1) the number of involved detectors $N_{\mathrm{SD}} \geq 4$, (2) zenith angle $\theta<55^{\circ}$, (3) $\chi^{2} / N_{\text {DOF }}<4$ where $N_{\text {DOF }}$ means the number of degree of freedom, (4) pointing direction uncertainty $\sigma_{\mathrm{PD}}<8^{\circ}$, and (5) $\sigma_{S 800} / S 800<0.5$.

\subsubsection{Quality Cut Efficiency and Energy Resolution}

Figure 5 shows the quality cut efficiency and the number of reconstructed events after the quality cuts (1)-(5) as a function of energy, which are estimated by the MC simulation. The energy resolution is defined as the fractional RMS of $E_{\text {rec }} / E_{\mathrm{thr}}$, where $E_{\text {rec }}$ and $E_{\mathrm{thr}}$ represents the reconstructed energy and thrown (true) energy, respectively. With all quality cuts applied, 


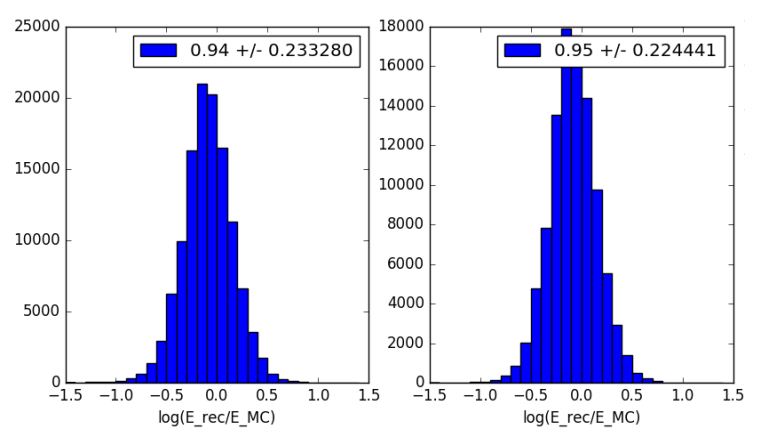

Figure 6: $E_{\mathrm{rec}} / E_{\mathrm{thr}}$ distribution with quality cut (1) (left) and (1)-(5) (right)

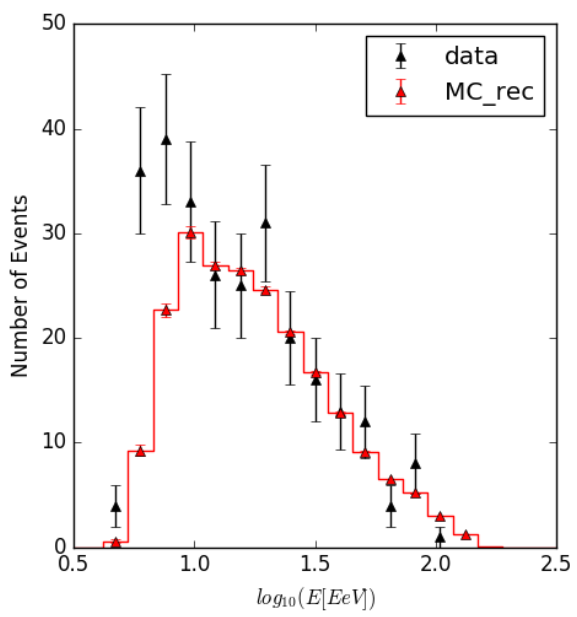

Figure 7: Energy distribution measured by the TAx4 SDs during the period from Nov 2019 to Oct 2020.

the energy resolution and angular resolution of the TAx4 SDs are estimated to be $22 \%$ and $2.0^{\circ}$, respectively above $57 \mathrm{EeV}$ assuming that all the SDs are available under the ideal condition as shown in Figure 6.

\subsection{Energy Distribution}

Figure 7 shows the reconstructed energy distribution, multiplying the number of events by the reconstruction efficiency in each energy bin, compared with that of the MC simulation. The cosmicray flux rapidly decreases in to $E^{-3}$, while the reconstruction efficiency increases as the cosmic-ray energy increases, because the higher energy cosmic ray has much more secondary particles in the air shower. Therefore, the reconstructed mode energy is approximately $10 \mathrm{EeV}$. The MC simulation is reasonable agreement with the experimental data in Figure 7.

\subsection{Energy Spectrum}

Figure 8 shows the preliminary energy spectrum above $10 \mathrm{EeV}$ measured by the TAx 4 SDs. The differential energy flux in each energy bin is calculated by

$$
J\left(E_{i}\right)=\frac{N\left(E_{i}\right)}{\Delta E_{i} \epsilon\left(E_{i}\right) \int A \Omega d t},
$$



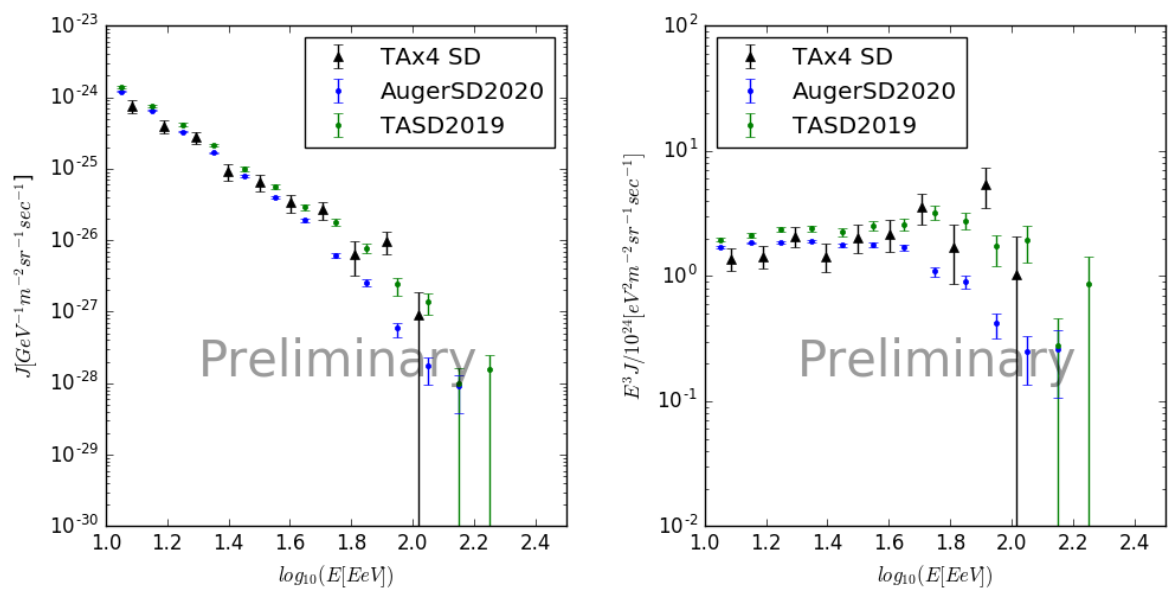

Figure 8: Preliminary energy spectrum measured by the TAx4 SDs together with the TA [7] and the Auger spectra [8]. The left and right panels show the differential spectrum and spectrum multiplied by $E^{3}$, respectively.

where $N\left(E_{i}\right)$ is the number of observed events, $\Delta E_{i}$ is the energy bin width, $\epsilon\left(E_{i}\right)$ is the efficiency in $i$-th energy bin, and $\int A \Omega d t$ is the exposure. Here, exposure is simplified as $A \Omega T$, where $A$ is the detection area, $\Omega=2.679 \mathrm{sr}$ is the solid angle of the field of view, and $T=1$ year is the observation time. The detection area $A$ is simply calculated to be used area, $2.08 \mathrm{~km} \times 2.08 \mathrm{~km} \times 137$. The measured spectrum above $10 \mathrm{EeV}$ by the TAx 4 SDs is overall consistent with the previous works.

\section{Summary}

The TAx 4 experiment, which currently consists of 257 SDs and 2 FD stations, had been built in Utah, USA in 2019. In order to improve energy resolution and the angular resolution, we have developed the air shower reconstruction method of the TAx4 SDs using the experimental data and the MC simulation. The energy resolution and the angular resolution of the TAx 4 SDs are estimated to be $22 \%$ and $2.0^{\circ}$, respectively, above $57 \mathrm{EeV}$ assuming that all the SDs are available under the ideal condition. The preliminary energy spectrum above $10 \mathrm{EeV}$ measured by the TAx 4 SDs for 1 year is overall consistent with the previous works.

\section{Acknowledgements}

The Telescope Array experiment is supported by the Japan Society for the Promotion of Science(JSPS) through Grants-in-Aid for Priority Area 431, for Specially Promoted Research JP21000002, for Scientific Research (S) JP19104006, for Specially Promoted Research JP15H05693, for Scientific Research (S) JP15H05741, for Science Research (A) JP18H03705, for Young Scientists (A) JPH26707011, and for Fostering Joint International Research (B) JP19KK0074, by the joint research program of the Institute for Cosmic Ray Research (ICRR), The University of Tokyo; by the Pioneering Program of RIKEN for the Evolution of Matter in the Universe (r-EMU); by the U.S. National Science Foundation awards PHY-1404495, PHY-1404502, PHY-1607727, PHY-1712517, PHY-1806797 and PHY-2012934; by the National Research Foundation of Korea 
(2017K1A4A3015188, 2020R1A2C1008230, \& 2020R1A2C2102800) ; by the Ministry of Science and Higher Education of the Russian Federation under the contract 075-15-2020-778, RFBR grant 20-02-00625a (INR), IISN project No. 4.4501.18, and Belgian Science Policy under IUAP VII/37 (ULB). This work was partially supported by the grants of The joint research program of the Institute for Space-Earth Environmental Research, Nagoya University and Inter-University Research Program of the Institute for Cosmic Ray Research of University of Tokyo. The foundations of Dr. Ezekiel R. and Edna Wattis Dumke, Willard L. Eccles, and George S. and Dolores Doré Eccles all helped with generous donations. The State of Utah supported the project through its Economic Development Board, and the University of Utah through the Office of the Vice President for Research. The experimental site became available through the cooperation of the Utah School and Institutional Trust Lands Administration (SITLA), U.S. Bureau of Land Management (BLM), and the U.S. Air Force. We appreciate the assistance of the State of Utah and Fillmore offices of the BLM in crafting the Plan of Development for the site. Patrick A. Shea assisted the collaboration with valuable advice and supported the collaboration's efforts. The people and the officials of Millard County, Utah have been a source of steadfast and warm support for our work which we greatly appreciate. We are indebted to the Millard County Road Department for their efforts to maintain and clear the roads which get us to our sites. We gratefully acknowledge the contribution from the technical staffs of our home institutions. An allocation of computer time from the Center for High Performance Computing at the University of Utah is gratefully acknowledged.

\section{References}

[1] R. U. Abbasi et al., Indications of Intermediate-scale Anisotropy of Cosmic Rays with Energy Greater Than $57 \mathrm{EeV}$ in the Northern Sky Measured with the Surface Detector of the Telescope Array Experiment, ApJ 790, L21 (2014).

[2] E. Kido et al., Status and Prospects of the TAx4 Experiment, EPJ Web Conf. 210, 06001 (2019).

[3] S. Yoshida et al., Lateral distribution of charged particles in giant air showers above $1 \mathrm{EeV}$ observed by AGASA, Journal of Physics G: Nuclear and Particle Physics 20, 651 (1994).

[4] D. Heck, J. Knapp, J. N. Capdevielle, G. Schatz and T. Thouw, et al., Corsika: A monte carlo code to simulate extensive air showers, Report fzka, 6019 (1998).

[5] S. Ostapchenko, QGSJET-II: physics, recent improvements, and results for air showers, EPJ Web Conf. 52, 02001 (2013).

[6] R. U. Abbasi et al., The Cosmic-Ray Energy Spectrum Observed with the Surface Detector of the Telescope Array Experiment, ApJ 768, L1 (2013).

[7] D. Ivanov et al., Energy Spectrum Measured by the Telescope Array, PoS(ICRC2019) 358, 298 (2019).

[8] A. Aab et al., Measurement of the cosmic-ray energy spectrum above $2.5 \times 10^{18} \mathrm{eV}$ using the Pierre Auger Observatory, PRD 102, 062005 (2020). 


\section{Full Authors List: Telescope Array Collaboration}

R.U. Abbasi ${ }^{1,2}$, T. Abu-Zayyad ${ }^{1,2}$, M. Allen ${ }^{2}$, Y. Arai $^{3}$, R. Arimura ${ }^{3}$, E. Barcikowski ${ }^{2}$, J.W. Belz ${ }^{2}$, D.R. Bergman ${ }^{2}$, S.A. Blake ${ }^{2}$, I. Buckland ${ }^{2}$, R. $\mathrm{Cady}^{2}$, B.G. Cheon ${ }^{4}$, J. Chiba ${ }^{5}$, M. Chikawa ${ }^{6}$, T. Fujii ${ }^{7}$, K. Fujisue ${ }^{6}$, K. Fujita ${ }^{3}$, R. Fujiwara ${ }^{3}$, M. Fukushima ${ }^{6}$, R. Fukushima ${ }^{3}$, G. Furlich ${ }^{2}$, R. Gonzalez ${ }^{2}$, W. Hanlon ${ }^{2}$, M. Hayashi ${ }^{8}$, N. Hayashida ${ }^{9}$, K. Hibino ${ }^{9}$, R. Higuchi ${ }^{6}$, K. Honda ${ }^{10}$, D. Ikeda ${ }^{9}$, T. Inadomi ${ }^{11}$, N. Inoue ${ }^{12}$, T. Ishii ${ }^{10}$, H. Ito $^{13}$, D. Ivanov ${ }^{2}$, H. Iwakura ${ }^{11}$, A. Iwasaki ${ }^{3}$, H.M. Jeong ${ }^{14}$, S. Jeong ${ }^{14}$, C.C.H. Jui ${ }^{2}$, K. Kadota ${ }^{15}$, F. Kakimoto ${ }^{9}$, O. Kalashev ${ }^{16}$, K. Kasahara ${ }^{17}$, S. Kasami ${ }^{18}$, H. Kawai ${ }^{19}$, S. Kawakami ${ }^{3}$, S. Kawana ${ }^{12}$, K. Kawata ${ }^{6}$, I. Kharuk ${ }^{16}$, E. Kido ${ }^{13}$, H.B. Kim ${ }^{4}$, J.H. Kim ${ }^{2}$, J.H. Kim ${ }^{2}$, M.H. Kim ${ }^{14}$, S.W. Kim ${ }^{14}$, Y. Kimura ${ }^{3}$, S. Kishigami ${ }^{3}$, Y. Kubota ${ }^{11}$, S. Kurisu ${ }^{11}$, V. Kuzmin ${ }^{16}$, M. Kuznetsov ${ }^{16,20}$, Y.J. Kwon ${ }^{21}$, K.H. Lee ${ }^{14}$, B. Lubsandorzhiev ${ }^{16}$, J.P. Lundquist ${ }^{2,22}$, K. Machida ${ }^{10}$, H. Matsumiya ${ }^{3}$, T. Matsuyama ${ }^{3}$, J.N. Matthews ${ }^{2}$, R. Mayta ${ }^{3}$, M. Minamino ${ }^{3}$, K. Mukai ${ }^{10}$, I. Myers ${ }^{2}$, S. Nagataki ${ }^{13}$, K. Nakai ${ }^{3}$, R. Nakamura ${ }^{11}$, T. Nakamura ${ }^{23}$, T. Nakamura ${ }^{11}$, Y. Nakamura ${ }^{11}$, A. Nakazawa ${ }^{11}$, E. Nishio ${ }^{18}$, T. Nonaka ${ }^{6}$, H. Oda ${ }^{3}$, S. Ogio $^{3,24}$, M. Ohnishi ${ }^{6}$, H. Ohoka ${ }^{6}$, Y. Oku ${ }^{18}$, T. Okuda ${ }^{25}$, Y. Omura ${ }^{3}$, M. Ono ${ }^{13}$, R. Onogi ${ }^{3}$, A. Oshima ${ }^{3}$, S. Ozawa ${ }^{26}$, I.H. Park ${ }^{14}$, M. Potts ${ }^{2}$, M.S. Pshirkov ${ }^{16,27}$, J. Remington ${ }^{2}$, D.C. Rodriguez ${ }^{2}$, G.I. Rubtsov ${ }^{16}$, D. Ryu ${ }^{28}$, H. Sagawa ${ }^{6}$, R. Sahara ${ }^{3}$, Y. Saito ${ }^{11}$, N. Sakaki ${ }^{6}$, T. Sako ${ }^{6}$, N. Sakurai ${ }^{3}$, K. Sano ${ }^{11}$, K. Sato ${ }^{3}$, T. Seki ${ }^{11}$, K. Sekino ${ }^{6}$, P.D. Shah ${ }^{2}$, Y. Shibasaki ${ }^{11}$, F. Shibata ${ }^{10}$, N. Shibata ${ }^{18}$, T. Shibata ${ }^{6}$, H. Shimodaira ${ }^{6}$, B.K. Shin ${ }^{28}$, H.S. Shin ${ }^{6}$, D. Shinto ${ }^{18}$, J.D. Smith ${ }^{2}$, P. Sokolsky ${ }^{2}$, N. Sone ${ }^{11}$, B.T. Stokes ${ }^{2}$, T.A. Stroman ${ }^{2}$, Y. Takagi ${ }^{3}$, Y. Takahashi ${ }^{3}$, M. Takamura $^{5}$, M. Takeda ${ }^{6}$, R. Takeishi ${ }^{6}$, A. Taketa $^{29}$, M. Takita ${ }^{6}$, Y. Tameda ${ }^{18}$, H. Tanaka ${ }^{3}$, K. Tanaka ${ }^{30}$, M. Tanaka ${ }^{31}$, Y. Tanoue ${ }^{3}$, S.B. Thomas ${ }^{2}$, G.B. Thomson ${ }^{2}$, P. Tinyakov ${ }^{16,20}{ }^{\text {I. } \text { Tkachev }^{16}, \text { H. Tokuno }}{ }^{32}$, T. Tomida ${ }^{11}$, S. Troitsky ${ }^{16}$, R. Tsuda ${ }^{3}$, Y. Tsunesada ${ }^{3,24}$, Y. Uchihori ${ }^{33}$, S. Udo ${ }^{9}$, T. Uehama ${ }^{11}$, F. Urban ${ }^{34}$, T. Wong ${ }^{2}$, K. Yada $^{6}$, M. Yamamoto ${ }^{11}$, K. Yamazaki ${ }^{9}$, J. Yang ${ }^{35}$, K. Yashiro ${ }^{5}$, F. Yoshida ${ }^{18}$, Y. Yoshioka ${ }^{11}$, Y. Zhezher ${ }^{6,16}$, and Z. Zundel ${ }^{2}$

${ }^{1}$ Department of Physics, Loyola University Chicago, Chicago, Illinois, USA

${ }^{2}$ High Energy Astrophysics Institute and Department of Physics and Astronomy, University of Utah, Salt Lake City, Utah, USA

${ }^{3}$ Graduate School of Science, Osaka City University, Osaka, Osaka, Japan

${ }^{4}$ Department of Physics and The Research Institute of Natural Science, Hanyang University, Seongdong-gu, Seoul, Korea

${ }^{5}$ Department of Physics, Tokyo University of Science, Noda, Chiba, Japan

${ }^{6}$ Institute for Cosmic Ray Research, University of Tokyo, Kashiwa, Chiba, Japan

7 The Hakubi Center for Advanced Research and Graduate School of Science, Kyoto University, Kitashirakawa-Oiwakecho, Sakyo-ku, Kyoto, Japan

${ }^{8}$ Information Engineering Graduate School of Science and Technology, Shinshu University, Nagano, Nagano, Japan

${ }^{9}$ Faculty of Engineering, Kanagawa University, Yokohama, Kanagawa, Japan

${ }^{10}$ Interdisciplinary Graduate School of Medicine and Engineering, University of Yamanashi, Kofu, Yamanashi, Japan

${ }^{11}$ Academic Assembly School of Science and Technology Institute of Engineering, Shinshu University, Nagano, Nagano, Japan

12 The Graduate School of Science and Engineering, Saitama University, Saitama, Saitama, Japan 
${ }^{13}$ Astrophysical Big Bang Laboratory, RIKEN, Wako, Saitama, Japan

${ }^{14}$ Department of Physics, SungKyunKwan University, Jang-an-gu, Suwon, Korea

${ }^{15}$ Department of Physics, Tokyo City University, Setagaya-ku, Tokyo, Japan

${ }^{16}$ Institute for Nuclear Research of the Russian Academy of Sciences, Moscow, Russia

${ }^{17}$ Faculty of Systems Engineering and Science, Shibaura Institute of Technology, Minato-ku, Tokyo, Japan

${ }^{18}$ Department of Engineering Science, Faculty of Engineering, Osaka Electro-Communication University, Neyagawa-shi, Osaka, Japan

${ }^{19}$ Department of Physics, Chiba University, Chiba, Chiba, Japan

${ }^{20}$ Service de Physique Théorique, Université Libre de Bruxelles, Brussels, Belgium

${ }^{21}$ Department of Physics, Yonsei University, Seodaemun-gu, Seoul, Korea

${ }^{22}$ Center for Astrophysics and Cosmology, University of Nova Gorica, Nova Gorica, Slovenia

${ }^{23}$ Faculty of Science, Kochi University, Kochi, Kochi, Japan

${ }^{24}$ Nambu Yoichiro Institute of Theoretical and Experimental Physics, Osaka City University, Osaka, Osaka, Japan

${ }^{25}$ Department of Physical Sciences, Ritsumeikan University, Kusatsu, Shiga, Japan

${ }^{26}$ Quantum ICT Advanced Development Center, National Institute for Information and Communications Technology, Koganei, Tokyo, Japan

${ }^{27}$ Sternberg Astronomical Institute, Moscow M.V. Lomonosov State University, Moscow, Russia

28 Department of Physics, School of Natural Sciences, Ulsan National Institute of Science and Technology, UNIST-gil, Ulsan, Korea

${ }^{29}$ Earthquake Research Institute, University of Tokyo, Bunkyo-ku, Tokyo, Japan

${ }^{30}$ Graduate School of Information Sciences, Hiroshima City University, Hiroshima, Hiroshima, Japan

${ }^{31}$ Institute of Particle and Nuclear Studies, KEK, Tsukuba, Ibaraki, Japan

${ }^{32}$ Graduate School of Science and Engineering, Tokyo Institute of Technology, Meguro, Tokyo, Japan

${ }^{33}$ Department of Research Planning and Promotion, Quantum Medical Science Directorate, National Institutes for Quantum and Radiological Science and Technology, Chiba, Chiba, Japan

${ }^{34}$ CEICO, Institute of Physics, Czech Academy of Sciences, Prague, Czech Republic

${ }^{35}$ Department of Physics and Institute for the Early Universe, Ewha Womans University, Seodaaemungu, Seoul, Korea 